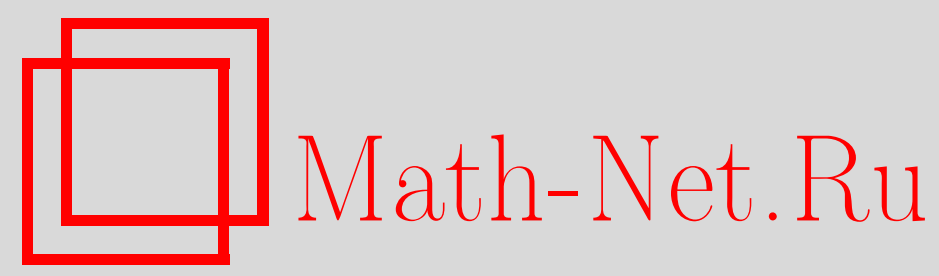

В. И. Масол, Теорема о предельном распределении числа ложных решений системы нелинейных случайных булевых уравнений, Теория вероятн. и ее примен., 1998, том 43, выпуск $1,41-56$

DOI: https://doi.org/10.4213/tvp822

Использование Общероссийского математического портала Math-Net.Ru подразумевает, что вы прочитали и согласны с пользовательским соглашением http://www.mathnet.ru/rus/agreement

Параметры загрузки:

IP : 18.208 .226 .222

26 апреля 2023 г., 12:01:02

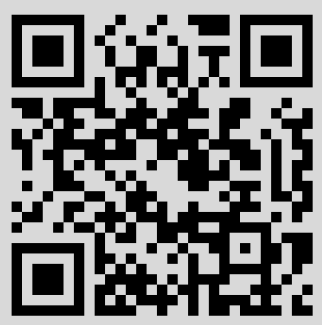


(c) $1998 \Gamma$.

МАСОЛ В. И.

\section{ТЕОРЕМА О ПРЕДЕЛЬНОМ РАСПРЕДЕЛЕНИИ ЧИСЛА ЛОЖНЫХ РЕІЕНИЙ СИСТЕМЫ НЕЛИНЕЙНЫХ СЛУЧАЙНЫХ БУЛЕВЫХ УРАВНЕНИЙ}

Доказана теорема о пуассоновском с параметром $2^{m}$ предельном $(n \rightarrow \infty)$ распределении числа ложных решений заведомо совместной системы нелинейных случайных булевых уравнений со стохастически независимыми коэффициентами. Среди предположений теоремы, в частности, следуюшие: распределения коэффициентов изменяются в некоторой окрестности точки $\frac{1}{2}$; число $n$ неизвестных и число $N$ уравнений системы отличаются на константу $m$ при $n \rightarrow \infty$; система имеет решение с числом единиц $\rho(n), \rho(n) \rightarrow \infty$ при $n \rightarrow \infty$.

Ключевые слова и фразы: число ложных решений, распределение Пуассона, нелинейные случайные булевы уравнения.

1. Постановка задачи. Формулировка теоремы. Рассмотрим над полем $\mathrm{GF}(2)$ систему уравнений

$$
\sum_{k=1}^{g_{i}(n)} \sum_{1 \leqslant j_{1}<\cdots<j_{k} \leqslant n} a_{j_{1} \cdots j_{k}}^{(i)} x_{j_{1}} \cdots x_{j_{k}}=b_{i}, \quad i=1, \ldots, N
$$

удовлетворяющую следующему условию (А).

У с л о в и е (A). 1) Коэффишиенты $a_{j_{1} \cdots j_{k}}^{(i)}, 1 \leqslant j_{1}<\cdots<j_{k} \leqslant n$, $k=1, \ldots, g_{i}(n), i=1, \ldots, N$, есть независимые случайные величины, принимающие значение 1 с вероятностью $\mathbf{P}\left\{a_{j_{1} \cdots j_{k}}^{(i)}=1\right\}=p_{i k}$ и значение 0 с вероятностью $\mathbf{P}\left\{a_{j_{1} \cdots j_{k}}^{(i)}=0\right\}=1-p_{i k}$.

2) Элементы $b_{i}, i=1, \ldots, N$ представляют собой результат подстановки в левую часть системы (1.1) фиксированного $n$-мерного вектора $\bar{x}^{0}$, имеюшего $\rho(n)(n-\rho(n))$ компонент, равных единице (нулю).

3) Функщии $g_{i}(n), i=1, \ldots, N$, - неслучайные, $g_{i}(n) \in\{2, \ldots, n\}$, $i=1, \ldots, N$.

Обзор результатов для линейного $\left(g_{i}(n) \equiv 1, i=1, \ldots, N\right)$ варианта (1.1) дан в [1]. В работах [2], [3] изучено распределение числа реше-

*Киевский университет им. Т. Шевченко, мех.-матем. ф-т, кафедра теории вероятн. и матем. статистики, ул. Владимирская, 64, 252601 Киев, Украина. 
ний системы (1.1) при специальных ограничениях на индексы $1, \ldots, n$ неизвестных $x_{1}, \ldots, x_{n}$.

Обозначим через $\nu_{n}$ число ложных решений (1.1), т.е. число решений системы (1.1), отличных от $\bar{x}^{0}$.

Теорема. Пусть выполняются условия (A),

$$
n-N=m, \quad n \rightarrow \infty, m=\text { const },-\infty<m<\infty
$$

для произвольного $i, i=1, \ldots, N$, существует пакое множество $T_{i}$, что при $n \rightarrow \infty$

$$
\begin{aligned}
T_{i} & \subseteq\left\{2, \ldots, g_{i}(n)\right\}, \quad T_{i} \neq \varnothing ; \\
\delta_{i t}(n) & \leqslant p_{i t} \leqslant 1-\delta_{i t}(n), \quad t \in T_{i}, i=1, \ldots, N
\end{aligned}
$$

найдется функчия $\varphi(n), \varphi(n) \leqslant n, n p u$ которой

$$
\sum_{i=1}^{N} \exp \left\{-2 \sum_{t \in T_{i}} \delta_{i t}(n) C_{f(n)}^{t}\right\} \rightarrow 0, \quad n \rightarrow \infty,
$$

где $f(n)$ принимает целые положительные значения, $f(n)=o(\varphi(n))$, $n \rightarrow \infty$, и существует такое $\varepsilon_{0}, \varepsilon_{0} \in(0,1)$, что для $0<\varepsilon \leqslant \varepsilon_{0}$

$$
\sum_{t=1}^{[\varepsilon \varphi(n)]} C_{t(n)}^{t} \leqslant \exp \{(\sigma(\varepsilon)+o(1)) \rho(n)\}, \quad n \rightarrow \infty
$$

$t(n) \in\{\rho(n), n-\rho(n)\}, \sigma(\varepsilon) \rightarrow 0$ nрu $\varepsilon \rightarrow 0$. Тогда для $k=0,1, \ldots$

$$
\lim _{n \rightarrow \infty} \mathbf{P}\left\{\nu_{n}=k\right\}=\frac{e^{-\lambda} \lambda^{k}}{k !},
$$

гдe $\lambda=2^{m}$.

Сформулированная теорема анонсирована в [4]. Там же приведены условия, достаточные для выполнения (1.3)-(1.6).

2. Вспомогательные утверждения. Пусть $W-$ совокупность всех непустых подмножеств множества $\Omega$, мощность которого равняется $|\Omega|=k, 1 \leqslant k<\infty$. Определим два подмножества $W_{\Delta}$ и $I_{s}$ множества $W$ :

$$
W_{\Delta} \subseteq W, \quad W_{\Delta}=\left\{\omega_{1}, \ldots, \omega_{\Delta}\right\}, \quad\left|W_{\Delta}\right|=\Delta, \quad \Delta \geqslant 1, \omega_{i} \neq \omega_{j}
$$

для $i \neq j, i, j \in\{1, \ldots, \Delta\}$;

$$
I_{s} \subseteq W, \quad I_{s}=\left\{m_{1}, \ldots, m_{s}\right\}, \quad\left|I_{s}\right|=s, \quad s \geqslant 0, m_{i} \neq m_{j}
$$

для $i \neq j, i, j \in\{1, \ldots, s\}$.

Утверждение 2.1. Пусть

$$
\begin{aligned}
\left|m_{i} \cap m_{j}\right| & \equiv 0(\bmod 2), \quad i=1, \ldots, s, j=1, \ldots, \Delta ; \\
\Delta & \in\left[2^{r-1}, 2^{r}-1\right], \quad 1 \leqslant r \leqslant k .
\end{aligned}
$$


Toгda

$$
s \leqslant 2^{k-r}-1
$$

Д о к а з а т е л с т в о. Если $s=0$, то, очевидно, (2.3) выполняется. Пусть $s>0$. Обозначим $L_{\alpha_{1}, \ldots, \alpha_{z}}$ совокупность всех элементов, принадлежаших $\omega_{\alpha_{1}}, \ldots, \omega_{\alpha_{z}},\left|L_{\alpha_{1}, \ldots, \alpha_{z}}\right|=l_{\alpha_{1}, \ldots, \alpha_{z}}$, где $1 \leqslant \alpha_{1}<\cdots<$ $\alpha_{z} \leqslant \Delta, z=1, \ldots, \Delta$. Обозначим $\varphi$ общее количество положительных (непустых) элементов множества $\left\{l_{\alpha_{1}, \ldots, \alpha_{z}}: 1 \leqslant \alpha_{1}<\cdots<\alpha_{z} \leqslant \Delta, z=\right.$ $1, \ldots, \Delta\}\left(\left\{L_{\alpha_{1}, \ldots, \alpha_{z}}: 1 \leqslant \alpha_{1}<\cdots<\alpha_{z} \leqslant \Delta, z=1, \ldots, \Delta\right\}\right)$.

Определим вектор $\bar{x}(v), \bar{x}(v)=\left\{x_{1}(v), \ldots, x_{\varphi}(v)\right\}, v \in W$, следующим правилом (II):

$$
x_{t}(v)=\left\{\begin{array}{ll}
1, & \text { если }\left|v \cap L_{k_{t}}\right| \equiv 1(\bmod 2), \\
0, & \text { если }\left|v \cap L_{k_{t}}\right| \equiv 0(\bmod 2),
\end{array} \quad t=1, \ldots, \varphi,\right.
$$

где $k_{t}, t=1, \ldots, \varphi,-$ попарно различные элементы множества $\left\{\left\{\alpha_{1}, \ldots\right.\right.$, $\left.\left.\alpha_{z}\right\}: 1 \leqslant \alpha_{1}<\cdots<\alpha_{z} \leqslant \Delta, z=1, \ldots, \Delta\right\}$, каждому из которых соответствует положительное (непустое) $l_{k_{i}}\left(L_{k_{i}}\right)$.

С помощью правила (П) для произвольного $\widetilde{m}, \tilde{m} \in I_{s}$, можно построить вектор $\bar{x}(\tilde{m})$, который является решением однородной системы линейных уравнений

$$
\sum_{t=1}^{\varphi} a_{q}^{(t)} x_{t}=0, \quad q=1, \ldots, \Delta
$$

над полем $\mathrm{GF}(2)$, где коэффициент $a_{q}^{(t)}=\left\{1\right.$, если $q \in k_{t} ; 0$, если $\left.q \notin k_{t}\right\}$, $t=1, \ldots, \varphi, q=1, \ldots, \Delta$. (В дальнейшем матрицу $A, A=\left\|a_{q}^{(t)}\right\|_{q=1, \ldots, \Delta}^{t=1, \ldots, \varphi}$, будем называть матрицей, построенной на множестве $W_{\Delta}$.) $\mathrm{B}$ самом деле, из включения $\tilde{m} \in I_{s}$ следует, в силу (2.1), что $\left|\widetilde{m} \cap \omega_{j}\right| \equiv 0$ $(\bmod 2), j=1, \ldots, \Delta$, но $\omega_{j}=\cup_{t=1}^{\varphi} L_{k_{t}}^{(j)}$, где $L_{k_{t}}^{(j)}=\left\{L_{k_{t}}\right.$, если $a_{j}^{(t)}=1$; $\varnothing$, если $\left.a_{j}^{(t)}=0\right\}$, значит,

$$
\left|\tilde{m} \cap \omega_{j}\right|=\sum_{t=1}^{\varphi}\left|\tilde{m} \cap L_{k_{t}}^{(j)}\right|, \quad j=1, \ldots, \Delta,
$$

поэтому $a_{j}^{(1)} x_{1}(\tilde{\tilde{m}}) \oplus \cdots \oplus a_{j}^{(\varphi)} x_{\varphi}(\widetilde{m})=0, j=1, \ldots, \Delta$, где символ $\oplus$ обозначает сложение по модулю 2.

Для совокупности всех элементов множества $I_{s}$, соответствуюших построенному с помощью правила (II) решению $\bar{x}(\tilde{m})$ системы $(2.4)$, где $\tilde{m} \in I_{s}$, примем запись $M(\widetilde{m})$, т.е. $M(\widetilde{m}) \subseteq I_{s}, M(\widetilde{m})=\left\{v_{1}, \ldots, v_{\mu}\right\}, v_{i} \neq$ $v_{j}$ для $i, j \in\{1, \ldots, \mu\}, i \neq j, \bar{x}\left(v_{t}\right)=\bar{x}(\widetilde{m})$ для $t=1, \ldots, \mu, \bar{x}(v) \neq \bar{x}(\widetilde{m})$ для $v \in I_{s} \backslash M(\tilde{m})$. Пусть вектор $\bar{x}(\tilde{m})$ содержит $\varkappa, 0 \leqslant \varkappa \leqslant \varphi$, единичных компонент, расположенных на местах с номерами $i_{1}, \ldots, i_{\varkappa}$, и $\varphi-\varkappa$ нулевых компонент, расположенных на местах с номерами $j_{1}, \ldots, j_{\varphi-\varkappa}$. 
Тогда, очевидно, мощность $\mu$ множества $M(\tilde{m})$ удовлетворяет неравенству

$$
\mu \leqslant 2^{k-\dot{s}_{1}+s_{2}+s_{3}}-\chi\{\dot{\varkappa}=0\},
$$

где $s_{1}=\sum_{t=1}^{\varphi} l_{k_{t}}, 2^{k-s_{1}}-$ количество всех подмножеств множества $\Omega \backslash\left\{\bigcup_{t=1}^{\varphi} L_{k_{t}}\right\} ; s_{2}=\sum_{i \in\left\{i_{1}, \ldots, i_{\varkappa}\right\}}\left(l_{k_{i}}-1\right), 2^{l_{k_{i}}-1}-$ количество всех подмножеств множества $L_{k_{i}}, i \in\left\{i_{1}, \ldots, i_{\varkappa}\right\}$, каждое из которых содержит нечетное число элементов из $\Omega ; s_{3}=\sum_{j \in\left\{j_{1}, \ldots, j_{\varphi-x}\right\}}\left(l_{k_{j}}-1\right), 2^{l_{k_{j}}-1}-$ количество всех подможеств множества $L_{k_{j}}, j \in\left\{j_{1}, \ldots, j_{\varphi-\varkappa}\right\}$, каждое из которых содержит четное число элементов из $\Omega ; \chi\{\varkappa=0\}=\{1$, если $\varkappa=0 ; 0$, если $\varkappa \neq 0\}$. Из (2.5) и определений сумм $s_{1}, s_{2}, s_{3}$ следует, что $\mu \leqslant 2^{k-\varphi}-\chi\{\varkappa=0\}$. Итак, каждому $\widetilde{m} \in I_{s}$ соответствует построенное с помощью правила (II) решение системы (2.4), и число элементов из $I_{s}$, соответствуюших этому решению, не превосходит $2^{k-\varphi}-\chi\{\varkappa=0\}$. Очевидно, что

$$
s \leqslant 2^{k-\varphi} R-1,
$$

где $R$ - число решений системы (2.4). В силу (2.2) ранг $f$ матрицы $A$ коэффициентов системы $(2.4)$, rang $(A)=f$, над полем $\mathrm{GF}(2)$ удовлетворяет неравенству $f \geqslant r$. Поэтому $R=2^{\varphi-f} \leqslant 2^{\varphi-r}$. С учетом (2.6) получаем (2.3). Утверждение 2.1 доказано.

3 а м е ч а н и е 2.2. Из доказательства утверждения 2.1 следует, что если имеют место (2.1), (2.2) и $f>r$, то $s<2^{k-r}-1$. Поэтому при выполнении условий $(2.1),(2.2)$ и $s=2^{k-r}-1,1 \leqslant r \leqslant k$, справедливо равенство $\operatorname{rang}(A)=r$.

Утверждение 2.2. Пусть $\Omega=\{1, \ldots, k\}, 3 \leqslant k<\infty$. Если $\partial \Omega я$ множеств $W_{\Delta}$ и $I_{s}$ выполняются условия (2.1),

$$
\begin{aligned}
\Delta & =2^{r}-1, \quad s=2^{k-\tau}-1, \quad 1 \leqslant r \leqslant k-2 ; \\
\left|\omega_{j}\right| & \geqslant 3, \quad j=1, \ldots, \Delta,
\end{aligned}
$$

то существует такое число $\alpha, \alpha \in\{1, \ldots, \Delta\}$, что для некоторых $m_{i_{\nu}}$, $m_{i_{\nu}} \in I_{s}, \nu=1,2,3$,

$$
\left|\omega_{\alpha} \cap m_{i_{\nu}}\right|=2, \quad \nu=1,2,3, \quad\left|\omega_{\alpha} \cap(a \cup b)\right|=3,
$$

ade $a \neq b, a, b \in\left\{m_{i_{\nu}}: \nu=1,2,3\right\}$.

Док а з а те ль с т в о. Пусть матрица $A$ построена на множестве $W_{\Delta}$. На основании условий $(2.1),(2.7)$ и замечания 2.1 заключаем, что $\operatorname{rang}(A)=r$. Обозначим символом $B$ матрицу, имеющую $\Delta$ строк и $k$ столбцов, $B=\left\|b_{q}^{(\varkappa)}\right\|_{q=1, \ldots, \Delta}^{\varkappa=1}$, где $b_{q}^{(\varkappa)}=\left\{1\right.$, если $\varkappa \in \omega_{q} ; 0$, если $\left.\varkappa \notin \omega_{q}\right\}$, $\varkappa=1, \ldots, k, q=1, \ldots, \Delta$. Используя определения матриц $A$ и $B$ и метод математической индукции по параметру $r, r \geqslant 1$, нетрудно установить, что над полем $\mathrm{GF}(2)$ выполняется $\operatorname{rang}(A)=\operatorname{rang}(B)=r$. Поскольку матрица $B$ имеет $\Delta=2^{\tau}-1$ ненулевых строк и $\operatorname{rang}(B)=r$, ее первые 
$r$ строк можно выбрать таким образом, чтобы они образовывали канонический базис $r$-мерного подпространства $k$-мерного пространства над $\mathrm{GF}(2)$. Тогда согласно правилу задания канонического базиса (один из вариантов изложения этого правила см., например, в [5, с. 219]) в первых $r$ строках матрицы $B$ существует единичная $(r \times r)$-матрица. Пусть столбцы с номерами $1<a_{1}<\cdots<a_{r} \leqslant k$ образуют указанную $(r \times r)$ матрищу. Применим построенный канонический базис к формированию множеств $m_{i_{1}}, m_{i_{2}}$ и $m_{i_{3}}$, удовлетворяющих (2.9). В качестве $\alpha$ выбираем 1, т.е. $\alpha=1$. В силу (2.8) в первой строке матрицы $B$ на позициях с номерами $j_{1}$ и $j_{2}$ расположены единицы, где $j_{1}$ и $j_{2}$ - некоторые целые числа, удовлетворяюшие соотношениям $1 \leqslant j_{1}<j_{2}<a_{1}$. Далее воспользуемся методом математической индукции. Пусть $\operatorname{rang}(B)=1$. Тогда в $m_{i_{1}}\left(m_{i_{2}} ; m_{i_{3}}\right)$ включим лишь следующие элементы: $u_{j_{1}}^{(1)}$ и $u_{j_{2}}^{(1)}$ $\left(u_{j_{1}}^{(1)}\right.$ и $u_{a_{1}}^{(1)} ; u_{j_{2}}^{(1)}$ и $\left.u_{a_{1}}^{(1)}\right)$, где $u_{j_{1}}^{(1)}, u_{j_{2}}^{(1)}$ и $u_{a_{1}}^{(1)}$ 一 элементы множества $\omega_{1}$ $\left(u_{j_{1}}^{(1)}, u_{j_{2}}^{(1)}, u_{a_{1}}^{(1)} \in \omega_{1}\right)$, соответствующие по построению единицам первой строки матрицы $B$, расположенным на позициях с номерами $j_{1}, j_{2}$ и $a_{1}$. Обоснуем индукционный шаг от $\operatorname{rang}(B)=r-1, r \geqslant 2, \mathrm{k} \operatorname{rang}(B)=r$. Если $\left|m_{i_{\nu}} \cap \omega_{r}\right| \equiv 0(\bmod 2)$, то множество $m_{i_{\nu}}$ оставляем без изменения, иначе дополняем множество $m_{i_{\nu}}$ элементом $u_{a_{r}}^{(r)}, u_{a_{r}}^{(r)} \in \omega_{r}$, который соответствует единище, расположенной в $r$-й строке матрицы $B$ на позиции с номером $a_{r}, \nu=1,2,3$. Ясно, что построенное таким образом множество $m_{i_{\nu}}, \nu=1,2,3$, удовлетворяет соотношениям $(2.9)$ и

$$
\left|m_{i_{\nu}} \cap \omega_{j}\right| \equiv 0(\bmod 2), \quad j=\{1, \ldots, \Delta\}, \nu=1,2,3 .
$$

Из (2.10) следует, что $m_{i_{\nu}} \in I_{s}, \nu=1,2,3$, так как в силу утверждения 2.1 и условия (2.7) множество $I_{s}$ содержит, очевидно, все совокупности $m_{1}, \ldots, m_{s}$, для которых выполняется (2.1).

Утверждение 2.2 доказано.

3 а м е ч а н и е 2.2. При $r=k-1$ и $s=1$ условие (2.8) не выполняется. Действительно, в $i$-й строке, $i \in\{1, \ldots, r\}$, канонического базиса на позициях с номерами $j>a_{i}$ стоят нули, а позиции с номерами $j<a_{i}$, $j \notin\left\{a_{1}, \ldots, a_{i-1}\right\}, i \in\{2, \ldots, r\}$, и $j<a_{1}$ могут быть заполнены элементами поля $\mathrm{GF}(2)$ произвольным образом. (Здесь сохранены обозначения, введенные при доказательстве утверждения 2.2.) Поэтому если $r=k-1$ и $s=1$, то существует такое значение параметра $j, j \in\{1, \ldots, \Delta\}$, что $\left|\omega_{j}\right|<3$.

В дальнейшем $\bar{x}^{1}, \ldots, \bar{x}^{k}-$ попарно различные $n$-мерные $(0,1)$-векторы, не совпадаюшие с $\bar{x}^{0}, \bar{x}^{\nu}=\left(x_{1}^{\nu}, \ldots, x_{n}^{\nu}\right), \nu=0, \ldots, k, 1 \leqslant k<\infty$. Для параметра $t, t \in\{1, \ldots, n\}$, набора $\left\{\alpha_{1}, \ldots, \alpha_{t}\right\}, 1 \leqslant \alpha_{1}<\cdots<\alpha_{t} \leqslant$ $n$, параметра $\nu, \nu \in\{1, \ldots, k\}$, и набора $\left\{u_{1}, \ldots, u_{\nu}\right\}, 1 \leqslant u_{1}<\cdots<u_{\nu} \leqslant$ $k$, рассмотрим множество

$$
m\left(\alpha_{1}, \ldots, \alpha_{t} ; u_{1}, \ldots, u_{\nu}\right)=\left\{x_{\alpha_{1}}^{u_{z}} \cdots x_{\alpha_{t}}^{u_{z}} \oplus x_{\alpha_{1}}^{0} \cdots x_{\alpha_{t}}^{0}: z=1, \ldots, \nu\right\} .
$$


Будем говорить, что $m\left(\alpha_{1}, \ldots, \alpha_{t} ; u_{1}, \ldots, u_{\nu}\right)$ обладает свойством $E$, если $x_{\alpha_{1}}^{u_{z}} \cdots x_{\alpha_{t}}^{u_{z}} \oplus x_{\alpha_{1}}^{0} \cdots x_{\alpha_{t}}^{0}=1, \quad z=1, \ldots, \nu$, и для произвольного $v, v \in\{1, \ldots, k\} \backslash\left\{u_{1}, \ldots, u_{\nu}\right\}, x_{\alpha_{1}}^{v} \cdots x_{\alpha_{t}}^{v} \oplus x_{\alpha_{1}}^{0} \cdots x_{\alpha_{t}}^{0}=0$. Обозначим через $\gamma_{t}^{\left\{u_{1}, \ldots, u_{\nu}\right\}}$ число всех попарно различных элементов множества $\left\{m\left(\alpha_{1}, \ldots, \alpha_{t} ; u_{1}, \ldots, u_{\nu}\right): 1 \leqslant \alpha_{1}<\cdots<\alpha_{t} \leqslant n\right\}$, обладаюших свойством $E$. Пусть для $1 \leqslant u_{1}<\cdots<u_{\nu} \leqslant k, \nu=1, \ldots, k, t=1, \ldots, n$

$$
\Gamma_{t, k}^{\left\{u_{1}, \ldots, u_{\nu}\right\}}=\sum_{\psi=1}^{\nu} 2^{-1}\left(1-(-1)^{\psi}\right) \sum_{M} \sum_{l=0}^{k-\nu} \sum_{N} \gamma_{t}^{\left\{\sigma_{1}, \ldots, \sigma_{\psi}, \mu_{1}, \ldots, \mu_{l}\right\}},
$$

где $M=\left\{1 \leqslant \sigma_{1}<\cdots<\sigma_{\psi} \leqslant k, \sigma_{z} \in\left\{u_{1}, \ldots, u_{\nu}\right\}, z=1, \ldots, \psi\right\}$, $N=\left\{1 \leqslant \mu_{1}<\cdots<\mu_{l} \leqslant k, \mu_{z} \notin\left\{u_{1}, \ldots, u_{\nu}\right\}, z=1, \ldots, l\right\}$.

Нас интересуют оценки суммы $\Gamma_{t, k}^{\left\{u_{1}, \ldots, u_{\nu}\right\}}$, введенной равенством (2.11), и отдельных ее слагаемых. Пусть $i_{\left\{u_{1}, \ldots, u_{s}\right\}}\left(j_{\left\{u_{1}, \ldots, u_{s}\right\}}\right)-$ число единиц (нулей), расположенных на тех и только тех позициях всех векторов $\bar{x}^{u_{1}}, \ldots, \bar{x}^{u_{s}}$, на которых у всех векторов $\bar{x}^{u_{s+1}}, \ldots, \bar{x}^{u_{k}}, \bar{x}^{0}$ расположены нули (единицы) $u_{1}, \ldots, u_{k} \in\{1, \ldots, k\}, u_{i} \neq u_{j}$ для $i \neq j$, $i, j \in\{1, \ldots, k\}, 1 \leqslant s \leqslant k$. Для совокупности $\left\{u_{1}, \ldots, u_{\nu}\right\}, 1 \leqslant u_{1}<\cdots<$ $u_{\nu} \leqslant k, \nu=1, \ldots, k$, положим

$$
\begin{aligned}
& I_{\left\{u_{1}, \ldots, u_{\nu}\right\}}=\left\{i_{\left\{\sigma_{1}, \ldots, \sigma_{\psi}, \mu_{1}, \ldots, \mu_{l}\right\}}: A(\psi, l, k)\right\}, \\
& J_{\left\{u_{1}, \ldots, u_{\nu}\right\}}=\left\{j_{\left\{\sigma_{1}, \ldots, \sigma_{\psi}, \mu_{1}, \ldots, \mu_{l}\right\}}: A(\psi, l, k)\right\},
\end{aligned}
$$

где $A(\psi, l, k)$-- сокращенная запись следующего набора ограничений: $1 \leqslant \sigma_{1}<\cdots<\sigma_{\psi} \leqslant k, \sigma_{z} \in\left\{u_{1}, \ldots, u_{\nu}\right\}, z=1, \ldots, \psi, \psi=1, \ldots, \nu, \psi \equiv$ $1(\bmod 2), 1 \leqslant \mu_{1}<\cdots<\mu_{l} \leqslant k, \mu_{1}, \ldots, \mu_{l} \notin\left\{u_{1}, \ldots, u_{\nu}\right\}, l=0, \ldots, k-\nu$. Пусть $J=\left\{j_{\left\{u_{1}, \ldots, u_{\nu}\right\}}: 1 \leqslant u_{1}<\cdots<u_{\nu} \leqslant k, \nu=1, \ldots, k\right\}$ и $s^{\prime}=\sum_{j \in J} j$.

Утверждение 2.3. Для $1 \leqslant u_{1}<\cdots<u_{\nu} \leqslant k, \nu \in\{1, \ldots, k\}, u$ $t \in\{1, \ldots, n\}$ выполняется неравенство

$$
\Gamma_{t, k}^{\left\{u_{1}, \ldots, u_{\nu}\right\}} \geqslant \sum_{(i, j) \in T}\left(C_{i}^{t}+C_{j}^{t}\right)
$$

гдe $T=I_{\left\{u_{1}, \ldots, u_{\nu}\right\}} \times J_{\left\{u_{1}, \ldots, u_{\nu}\right\}} ; e c \Omega u$

$$
\rho(n)-s^{\prime} \geqslant t
$$

mo

$$
\Gamma_{t, k}^{\left\{u_{1}, \ldots, u_{\nu}\right\}} \geqslant C_{\rho(n)-s^{\prime}}^{t-1} \sum_{(i, j) \in T}(i+j)
$$

Пусть $1 \leqslant u_{1}<\cdots<u_{\nu} \leqslant k, \nu \in\{3,4, \ldots, k\} ;\left\{\sigma_{1}, \sigma_{2}, \sigma_{3}\right\} \subseteq$ $\left\{u_{1}, \ldots, u_{\nu}\right\}, 1 \leqslant \sigma_{1}<\sigma_{2}<\sigma_{3} \leqslant k ;\left\{u_{1}, \ldots, u_{\nu}\right\} \cap\left\{\mu_{1}, \ldots, \mu_{l}\right\}=\varnothing$, $1 \leqslant \mu_{1}<\cdots<\mu_{l} \leqslant k, l=0, \ldots, k-\nu$. Ecлu

$$
j_{*} \geqslant t
$$


где $t-$ челое число, $t \geqslant 1, j_{*}=\min \left\{j_{a}, j_{b}\right\}, a=\widetilde{\sigma} \cup \widetilde{\mu}, b=\widehat{\sigma} \cup \widehat{\mu}$, $\tilde{\sigma} \cup \widehat{\sigma}=\left\{\sigma_{1}, \sigma_{2}, \sigma_{3}\right\}, \widetilde{\sigma} \neq \widehat{\sigma},|\widetilde{\sigma}|=|\widehat{\sigma}|=2, \widetilde{\mu} \cup \widehat{\mu}=\left\{\mu_{1}, \ldots, \mu_{l}\right\}$, mo

$$
\gamma_{t}^{\{a \cup b\}} \geqslant t^{-1} j_{*}\left(j^{*}-2^{-1}\left(j^{*}-1\right)\right) C_{\left(j^{*} / 2\right)+\left(3 j_{*} / 4\right)-(5 / 4)}^{t-2}
$$

zдe $j^{*}=\max \left\{j_{a}, j_{b}\right\}$.

Д ок аз а т е л с с в о. Используя определения чисел $\gamma_{t}^{\left\{u_{1}, \ldots, u_{\nu}\right\}}$, $i_{\left\{u_{1}, \ldots, u_{\nu}\right\}}$ и $j_{\left\{u_{1}, \ldots, u_{\nu}\right\}}$, находим следующие оценки для слагаемых из правой части (2.11):

$$
\begin{aligned}
\gamma_{t}^{\left\{\sigma_{1}, \ldots, \sigma_{\psi}, \mu_{1}, \ldots, \mu_{l}\right\}} \geqslant & C_{i_{\left\{\sigma_{1}, \ldots, \sigma_{\psi}, \mu_{1}, \ldots, \mu_{l}\right\}}^{t}}^{t}+C_{j_{\left\{\sigma_{1}, \ldots, \sigma_{\psi}, \mu_{1}, \ldots, \mu_{l}\right\}}^{t}}^{t}, \\
\gamma_{t}^{\left\{\sigma_{1}, \ldots, \sigma_{\psi}, \mu_{1}, \ldots, \mu_{l}\right\}} \geqslant & C_{i_{\left\{\sigma_{1}, \ldots, \sigma_{\psi}, \mu_{1}, \ldots, \mu_{l}\right\}}^{t}+\rho(n)-s^{\prime}}^{t} \\
& +C_{j_{\left\{\sigma_{1}, \ldots, \sigma_{\psi}, \mu_{1}, \ldots, \mu_{l}\right\}}^{t}+\rho(n)-s^{\prime}}^{t}-2 C_{\rho(n)-s^{\prime}}^{t}, \\
\gamma_{t}^{\{a \cup b\}} \geqslant & C_{j_{a}+j_{b}}^{t}-C_{j_{a}}^{t}-C_{j_{b}}^{t} .
\end{aligned}
$$

Из (2.11) и (2.17) немедленно следует (2.12). Оценивая правую часть (2.18) с помошью установленного в [6] неравенства

$$
C_{\alpha}^{t}-C_{\alpha-\beta}^{t} \geqslant \beta C_{\alpha-2-1(1+\beta)}^{t-1}
$$

где $\alpha, \beta$ и $t$ - целые положительные числа, $\alpha-\beta \geqslant t$, с учетом (2.11) и (2.13) легко получить соотношение (2.14). Далее, в силу (2.15) и (2.20), имеем:

$$
\begin{gathered}
C_{j^{*}+j_{*}}^{t}-C_{j *}^{t} \geqslant j_{*} C_{j^{*}+2^{-1}\left(j_{*}-1\right)}^{t-1}, \\
C_{j^{*}+2^{-1}\left(j_{*}-1\right)}^{t-1}-C_{j_{*}-1}^{t-1} \geqslant\left(j^{*}-2^{-1}\left(j_{*}-1\right)\right) C_{\left(j^{*} / 2\right)+\left(3 j_{*} / 4\right)-(5 / 4)}^{t-2}
\end{gathered}
$$

Последние два неравенства позволяют заключить, что

$$
C_{j^{*}+j_{*}}^{t}-C_{j_{*}}^{t}-C_{j_{*}}^{t} \geqslant t^{-1} j_{*}\left(j^{*}-2^{-1}\left(j_{*}-1\right)\right) C_{\left(j^{*} / 2\right)+\left(3 j_{*} / 4\right)-(5 / 4)}^{t-2} .
$$

Из последнего соотношения с учетом (2.19) непосредственно следует (2.16).

Утверждение 2.3 доказано.

Обозначим через $\mathbf{E} \nu_{n}^{[k]}, k$-й, $k \geqslant 0$, факториальный момент случайной величины $\nu_{n}$, т.е. $\mathbf{E} \nu_{n}^{[k]}=\mathbf{E} \nu_{n}\left(\nu_{n}-1\right) \cdots\left(\nu_{n}-k+1\right)$; положим $\mathbf{E} \nu_{n}^{[0]} \equiv 1$.

Утверждение 2.4. Если выполняется условие (A), по для $k \geqslant 1$

$$
\mathrm{E} \nu_{n}^{[k]}=2^{-k N} S(n, k ; Q),
$$

zde

$$
S(n, k ; Q)=\sum_{s=0}^{n-\rho(n)} \sum(n-\rho(n)) !\left((n-\rho(n)-s) ! \prod_{i \in I} i !\right)^{-1}
$$




$$
\begin{aligned}
& \times \sum_{\substack{s^{\prime}=0, s^{\prime}+s \geqslant 1}}^{\rho(n)} \sum^{\prime} \rho(n) !\left(\left(\rho(n)-s^{\prime}\right) ! \prod_{j \in J} j !\right)^{-1} Q \\
Q= & \prod_{i=1}^{N}\left(1+\sum_{\nu=1}^{k} \sum_{1 \leqslant u_{1}<\cdots<u_{\nu} \leqslant k} \prod_{t=1}^{g_{i}(n)}\left(1-2 p_{i t}\right)^{\left.\Gamma_{t_{1} k}^{\left\{u_{1}, \ldots, u_{\nu}\right\}}\right)}\right)
\end{aligned}
$$

суммирование $\sum\left(\Sigma^{\prime}\right)$ осуществляется по всем $i \in I(j \in J)$, аде $I=$ $\left\{i_{\left\{u_{1}, \ldots, u_{\nu}\right\}}: 1 \leqslant u_{1}<\cdots<u_{\nu} \leqslant k, \nu=1, \ldots, k\right\}$, таким, что

$$
\sum_{i \in I} i=s \quad\left(\sum_{j \in J} j=s^{\prime}\right)
$$

в равенстве (2.22) числа $i(i \in I), j$ ( $j \in J)$ удовлетворяют соотношениям

$$
\begin{aligned}
& \sum_{i \in I_{\{u\}}, j \in J_{\{u\}}}(i+j) \geqslant 1, \quad u=1, \ldots, k, \\
& \sum_{l=0}^{k-2} \sum_{1 \leqslant \mu_{1}<\cdots<\mu_{l} \leqslant k}\left(i_{\left\{u_{1}, \mu_{1}, \ldots, \mu_{l}\right\}}+j_{\left\{u_{1}, \mu_{1}, \ldots, \mu_{l}\right\}}+i_{\left\{u_{2}, \mu_{1}, \ldots, \mu_{l}\right\}}\right. \\
& \left.+j_{\left\{u_{2}, \mu_{1} \cdots \mu_{l}\right\}}\right) \geqslant 1, \quad 1 \leqslant u_{1}<u_{2} \leqslant k ;
\end{aligned}
$$

$\partial \Omega \boldsymbol{A} \Gamma_{t, k}^{\left\{u_{1}, \ldots, u_{\nu}\right\}}, 1 \leqslant u_{1}<\cdots<u_{\nu} \leqslant k, \nu=1, \ldots, k, t=1, \ldots, g_{i}(n)$, $i=1, \ldots, N$, справедливо (2.11).

Д о к а 3 а т е л ь с т в о утверждения 2.4 осуществляется аналогично доказательству теоремы 1 из [6]. (Заметим, что в [6] утверждение 2.4 установлено при дополнительном предположении: $g_{i}(n)=n$, $i=1, \ldots, N$.)

Положим

$$
\delta_{i}=\min \left\{\sum_{t \in T_{i}} \delta_{i t}(n) C_{r}^{t-1}, \frac{2(\ln n)}{\sqrt{\varepsilon}} n\right\}
$$

где

$$
i=1, \ldots, N, \quad r=[\varepsilon \varphi(n)], \quad 0<\varepsilon<1, \quad \varepsilon=\text { const }, \quad \varphi(n) \leqslant n .
$$

Утверждение 2.5. Если выполняются условия (1.2), (1.3) $u$

$$
\sum_{i=1}^{N} \exp \left\{-2 \sum_{t \in T_{i}} \delta_{i t}(n) C_{r}^{t}\right\} \longrightarrow 0, \quad n \rightarrow \infty
$$

mo

$$
\sqrt{\varepsilon} \sum_{i=1}^{N} \delta_{i}-\ln n \longrightarrow \infty, \quad n \rightarrow \infty
$$


Д о к а 3 а т е л ь с т в о. В силу (1.3) и (2.26) для $0<\varepsilon<1$ имеем:

$$
\begin{aligned}
& \sum_{i=1}^{N} \exp \left\{-2 \sum_{t \in T_{i}} \delta_{i t}(n) C_{r}^{t}\right\} \\
& \geqslant \sum_{i=1}^{N} \exp \left\{-\sqrt{\varepsilon} n \sum_{t \in T_{i}} \delta_{i t}(n) C_{r}^{t-1}\right\} \longrightarrow 0, \quad n \rightarrow \infty .
\end{aligned}
$$

Значит,

$$
\begin{aligned}
\sum_{i=1}^{N} e^{-\sqrt{\varepsilon} n \delta_{i}} \leqslant & \sum_{i=1}^{N} \exp \left\{-\sqrt{\varepsilon} n \sum_{t \in T_{i}} \delta_{i t}(n) C_{r}^{t-1}\right\} \\
& +\sum_{i=1}^{N} e^{-2 \ln n} \longrightarrow 0, \quad n \rightarrow \infty .
\end{aligned}
$$

Неравенство Йенсена для выпуклой функции дает оценку

$$
\sum_{i=1}^{N} e^{-\sqrt{\varepsilon} n \delta_{i}} \geqslant \exp \left\{(\ln N)-\frac{\sqrt{\varepsilon} n}{N} \sum_{i=1}^{N} \delta_{i}\right\} \longrightarrow 0, \quad n \rightarrow \infty,
$$

которая с учетом (1.2) доказывает утверждение 2.5 .

3. Доказательство теоремы. Покажем, что для $k=0,1,2, \ldots$

$$
\mathbf{E} \nu_{n}^{[k]} \rightarrow 2^{m k}, \quad n \rightarrow \infty .
$$

При $k=0$ соотношение (3.1) очевидно. Докажем (3.1) для $1 \leqslant k<\infty$. С этой целью выражение (2.21) для $\mathrm{E} \nu_{n}^{[k]}$ разобьем на конечное число слагаемых:

$$
\mathbf{E} \nu_{n}^{[k]}=2^{-k N} \sum_{\Delta \geqslant 0} S^{(\Delta)}(n, k ; Q),
$$

где $S^{(\Delta)}(n, k ; Q)$ отличается от $S(n, k ; Q)$ тем, что все $i$ и $j(i \in I, j \in J)$, участвуюшие в записи $S(n, k ; Q)$ согласно $(2.22)$, принимают лишь такие значения, что существуют в точности $\Delta$ попарно различных наборов $\omega_{\alpha}=\left\{u_{1}^{(\alpha)}, \ldots, u_{\xi_{\alpha}}^{(\alpha)}\right\}, 1 \leqslant u_{1}^{(\alpha)}<\cdots<u_{\xi_{\alpha}}^{(\alpha)} \leqslant k, \xi_{\alpha} \in\{1, \ldots, k\}, \alpha=$ $1, \ldots, \Delta$, для каждого из которых найдется такое $t^{(\alpha)} \in\{2, \ldots, r\}$, что выполняется неравенство

$$
\Gamma_{t^{(a)}, k}^{\omega_{a}}<C_{r}^{t^{(\alpha)}}
$$

и для наборов $\left\{v_{1}, \ldots, v_{\gamma}\right\}, 1 \leqslant v_{1}<\cdots<v_{\gamma} \leqslant k, \gamma=1, \ldots, k$, удовлетворяющих соотношению $\left\{v_{1}, \ldots, v_{\gamma}\right\} \neq \omega_{\alpha}, \alpha=1, \ldots, \Delta$, имеет место оценка

$$
\Gamma_{t, k}^{\left\{v_{1}, \ldots, v_{\gamma}\right\}} \geqslant C_{r}^{t}
$$


для всех $t \in\{2, \ldots, r\}$. Убедимся в том, что для $k=1,2, \ldots$

$$
2^{-k N} S^{(0)}(n, k ; Q) \longrightarrow 2^{m k}, \quad n \rightarrow \infty
$$

Предварительно заметим, что при достаточно малом $\varepsilon, \varepsilon>0$, по крайней мере одно из двух неравенств $i \geqslant r$ или $j \geqslant r$ может выполняться для всех $i \in I$ или всех $j \in J$. Но если $i \geqslant r$ и (или) $j \geqslant r$ для всех $i \in I$ и (или) всех $j \in J$, то в силу (2.12) ощенка (3.4) имеет место для всех наборов $\left\{v_{1}, \ldots, v_{\gamma}\right\}, 1 \leqslant v_{1}<\cdots<v_{\gamma} \leqslant k, \gamma=1, \ldots, k$, и $t \in\{2, \ldots, r\}$. Значит, равенство $\Delta=0$ может достигаться.

При $\Delta=0$ с учетом оценки (3.4) и условия (1.4) находим:

$$
\begin{gathered}
\prod_{i=1}^{N}\left(1-\left(2^{k}-1\right) \exp \left\{-2 \sum_{t \in T_{i}} \delta_{i t}(n) C_{r}^{t}\right\}\right) \leqslant Q \\
\leqslant \prod_{i=1}^{N}\left(1+\left(2^{k}-1\right) \exp \left\{-2 \sum_{t \in T_{i}} \delta_{i t}(n) C_{r}^{t}\right\}\right) .
\end{gathered}
$$

Из условия (1.5) и неравенства $r>f(n)$ следует, что

$$
\sum_{i=1}^{N} \exp \left\{-2 \sum_{t \in T_{i}} \delta_{i t}(n) C_{r}^{t}\right\} \longrightarrow 0, \quad n \rightarrow \infty .
$$

Используя $(3.6),(3.7)$ и соотношения $\ln (1+u)=u+O\left(u^{2}\right), u \rightarrow 0$,

$$
\max _{1 \leqslant i \leqslant N} \exp \left\{-2 \sum_{t \in T_{i}} \delta_{i t}(n) C_{r}^{t}\right\} \longrightarrow 0, \quad n \rightarrow \infty
$$

нетрудно получить, что

$$
Q \rightarrow 1, \quad n \rightarrow \infty
$$

Если для некоторого набора $\left\{u_{1}, \ldots, u_{\nu}\right\}, 1 \leqslant u_{1}<\cdots<u_{\nu} \leqslant k$, $\nu=1, \ldots, k$, и некоторого $t \in\{2, \ldots, r\}$

$$
\Gamma_{t, k}^{\left\{u_{1}, \ldots, u_{\nu}\right\}}<C_{r}^{t}
$$

то соотношение (2.12) позволяет заключить, что

$$
0 \leqslant i<r, \quad i \in I_{\left\{u_{1}, \ldots, u_{\nu}\right\}} ; \quad 0 \leqslant j<r, \quad j \in J_{\left\{u_{1}, \ldots, u_{\nu}\right\}} .
$$

Поэтому сумма $S^{(0)}(n, k ; 1)$ (здесь $S^{(\Delta)}(n, k ; 1)=S^{(\Delta)}(n, k ; Q)$ при условии $Q \equiv 1, \Delta \geqslant 0$; аналогично $S(n, k ; 1)=S(n, k ; Q)$ при условии $Q \equiv 1)$ равняется $S^{(0)}(n, k ; 1)=2^{n k}-\sigma_{0}$, где $\sigma_{0}$ отличается от $S(n, k ; 1)$ тем, что суммирование $\sum\left(\Sigma^{\prime}\right)$ (см. (2.22)) осуществляется по всем $i \in I(j \in J)$ таким, что выполняется (2.23), и по крайней мере один из элементов множества $\left\{\Gamma_{t, k}^{\left\{u_{1}, \ldots, u_{\nu}\right\}}: 1 \leqslant u_{1}<\cdots<u_{\nu} \leqslant k, \nu=1, \ldots, k, t=2, \ldots, r\right\}$ удовлетворяет (3.9). Поскольку при выполнении (3.9) имеет место (3.10), 
то в левой части каждого из равенств (2.23) найдется $2^{k-1}$ слагаемых, ограниченных величиной $r$. Следовательно,

$$
0 \leqslant \sigma_{0} \leqslant \exp \{(\sigma(\varepsilon)+o(1)) n\} 2^{n(k-1)},
$$

где $\sigma(\varepsilon)>0, \sigma(\varepsilon) \rightarrow 0$ при $\varepsilon \rightarrow 0$. Заметим, что было использовано, в частности, следующее легко проверяемое неравенство:

$$
\sum_{i=1}^{r} C_{n}^{i} \leqslant \exp \{(\sigma(\varepsilon)+o(1)) n\}, \quad n \rightarrow \infty .
$$

ИтаK,

$$
S^{(0)}(n, k ; 1)=2^{n k}(1+o(1)), \quad n \rightarrow \infty .
$$

Соотношения (1.2), (3.8) и (3.12) очевидно доказывают (3.5).

Покажем, что для $\Delta \geqslant 1$ и $k=1,2, \ldots$

$$
2^{-k N} S^{(\Delta)}(n, k ; Q) \longrightarrow 0, \quad n \rightarrow \infty
$$

Действительно, пусть $I=M_{1} \cup M_{2}$ и $J=\widetilde{M}_{1} \cup \widetilde{M}_{2}$, где

$$
M_{1}=\bigcup_{i \in I, i \notin I_{\omega_{\alpha}}, \alpha=1, \ldots, \Delta} i, \quad \widetilde{M}_{1}=\bigcup_{j \in J, j \notin J_{\omega_{\alpha}}, \alpha=1, \ldots, \Delta} j
$$

и $M_{2}=I \backslash M_{1}, \widetilde{M}_{2}=J \backslash \widetilde{M}_{1}$. Мощность множества $M_{1}\left(\widetilde{M}_{1}\right)$ обозначим $R_{1}\left(\widetilde{R}_{1}\right)$. Пусть $z$ - наименьшее целое число, для которого выполняется неравенство $\Delta \leqslant 2^{z}-1$. Ясно, что

$$
1 \leqslant z \leqslant k \text {. }
$$

На основании утверждения 2.1 получаем:

$$
R_{1} \leqslant 2^{k-z}-1 ; \quad \widetilde{R}_{1} \leqslant 2^{k-z}-1
$$

При выполнении (3.4) с учетом условия (1.4) имеем следующее неравенство для $Q$ из левой части (3.13):

$$
|Q| \leqslant 2^{2 N} \prod_{i=1}^{N}\left(1+2^{-z}\left(2^{k}-\Delta-1\right) \exp \left\{-2 \sum_{t \in T_{i}} \delta_{i t}(n) C_{r}^{t}\right\}\right)
$$

Далее, из (3.3), в силу (3.9) и (3.10), следует, что

$$
0 \leqslant i<r \quad(0 \leqslant j<r)
$$

для всех $i \in M_{2}\left(j \in \widetilde{M}_{2}\right)$. Используя $(1.6),(3.15),(3.17)$ и ограничение $\mathrm{Ч}_{0}$ :

$$
\rho(n)-r+1 \leqslant s^{\prime} \leqslant \rho(n)
$$

находим при $n \rightarrow \infty$

$$
S^{(\Delta)}(n, k ; 1) \leqslant \exp \{(\sigma(\varepsilon)+o(1)) \rho(n)\} 2^{n(k-z)}\left(1-2^{z-k}\right)^{\rho(n)} .
$$


Из (1.5) и (1.6) вытекает, что при $n \rightarrow \infty$

$$
\rho(n) \rightarrow \infty \text {. }
$$

С помошью (1.2), (3.7), (3.14), (3.16), (3.18) и (3.19) нетрудно получить (3.13) при ограничении $Ч_{0}$.

Пусть выполняются ограничения $\mathrm{Ч}_{1}$ :

$$
s^{\prime} \leqslant \rho(n)-r
$$

существует $i \in M_{2}$ и (или) существует $j \in \widetilde{M}_{2}$ такое, что $i \in\left(r / E_{n}, r\right]$ и (или) $j \in\left(r / E_{n}, r\right]$, где при $n \rightarrow \infty$

$$
E_{n} \rightarrow \infty, \quad E_{n}=o(\ln n) .
$$

При выполнении $\bigcup_{1}$ можно воспользоваться неравенством $(2.14)$ и установить

$$
\Gamma_{t, k}^{\omega_{a}} \geqslant C_{r}^{t-1} \frac{r}{E_{n}}
$$

для всех $t \in\{2, \ldots, r\}$ и некоторого $\alpha, \alpha=1, \ldots, \Delta$. С помощью (3.22) и (1.4) находим для $i, i=1, \ldots, N$, оценку

$$
\left|\prod_{t=1}^{g_{i}(n)}\left(1-2 p_{i t}\right)^{\Gamma_{t, k}^{\omega_{\alpha}}}\right| \leqslant \exp \left\{-2 \sum_{t \in T_{i}} \delta_{i t}(n) C_{r}^{t-1} \frac{r}{E_{n}}\right\}
$$

с учетом которой получаем

$$
\begin{gathered}
|Q| \leqslant 2^{z N} \exp \left\{-2^{-z}\left(N-\sum_{i=1}^{N} \exp \left\{-2 \sum_{t \in T_{i}} \delta_{i t}(n) C_{r}^{t-1} \frac{r}{E_{n}}\right\}-\left(2^{k}-\Delta-1\right)\right.\right. \\
\left.\left.\times \sum_{i=1}^{N} \exp \left\{-2 \sum_{t \in T_{i}} \delta_{i t}(n) C_{r}^{t}\right\}\right)\right\}
\end{gathered}
$$

В силу неравенства Гёльдера имеем соотношение

$$
\begin{aligned}
& \sum_{i=1}^{N} \exp \left\{-2 \sum_{t \in T_{i}} \delta_{i t}(n) C_{r}^{t-1} \frac{r}{E_{n}}\right\} \\
& \leqslant\left(\sum_{i=1}^{N} \exp \left\{-\sum_{t \in T_{i}} \delta_{i t}(n) C_{r}^{t-1} \varphi(n)\right\}\right)^{A_{n}} N^{1-A_{n}},
\end{aligned}
$$

где $A_{n}=2 \varepsilon / E_{n}$, использование которого совместно с (3.7) и неравенством

$$
\sum_{i=1}^{N} \exp \left\{-2 \sum_{t \in T_{i}} \delta_{i t}(n) C_{r}^{t}\right\} \geqslant \sum_{i=1}^{N} \exp \left\{-\sum_{t \in T_{i}} \delta_{i t}(n) C_{\tau}^{t-1} \varphi(n)\right\},
$$


справедливым на основании (1.3), позволяет прийти от (3.23) к оценке

$$
|Q| \leqslant 2^{z N} \exp \left\{-2^{-z}\left(N-N^{1-A_{n}}\right)+o(1)\right\}, \quad n \rightarrow \infty .
$$

Далее, нетрудно проверить, что при выполнении (3.15) и (3.17) в силу (3.11)

$$
S^{(\Delta)}(n, k ; 1) \leqslant 2^{n(k-z)} \exp \{(\sigma(\varepsilon)+o(1)) n\}, \quad n \rightarrow \infty .
$$

Соотношения $(3.21),(3.24),(3.25)$ и условие (1.2) дают возможность заключить, что при достаточно малом $\varepsilon>0$

$$
\begin{aligned}
& 2^{-k N}\left|S^{(\Delta)}(n, k ; Q)\right| \\
& \quad \leqslant \exp \left\{-2^{-z} N\left(1-N^{-A_{n}}-\sigma(\varepsilon)+o(1)\right)\right\} \rightarrow 0, \quad n \rightarrow \infty,
\end{aligned}
$$

т.е. (3.13) справедливо при ограничениях $\mathrm{\Psi}_{1}$.

Пусть выполняются ограничения $\mathrm{Ч}_{2}:(3.20)$; супествует $i \in M_{2}$ и (или) существует $j \in \widetilde{M}_{2}$ такое, что $\ddot{i} \in\left(r / \ln n, r / E_{n}\right]$ и (или) $j \in$ $\left(r / \ln n, r / E_{n}\right]$.

При выполнении Ч Ч $_{2}$ аналогично (3.24) (с тем лишь отличием, что в (3.24) вместо $A_{n}$ будет $\widetilde{A}_{n}=2 \varepsilon / \ln n$ ) получаем

$$
|Q| \leqslant 2^{z N} \exp \{-\sigma(\varepsilon) n\}, \quad n \rightarrow \infty .
$$

Если параметры $i$ и $j$ изменяются так, как указано в ограничениях $\Psi_{2}$, то, используя (3.15) и вариант неравенства (3.11), а именно,

$$
\sum_{i=0}^{n / E_{n}} C_{n}^{i} \leqslant \exp \{|o(n)|\}, \quad n \rightarrow \infty
$$

имеем

$$
S^{(\Delta)}(n, k ; 1) \leqslant 2^{n(k-z)} \exp \{|o(n)|\}, \quad n \rightarrow \infty .
$$

С помощью (3.26), (3.27) и условия (1.2) получаем (3.13) при ограничениях $\mathrm{Ч}_{2}$.

Пусть выполняется ограничение $\mathrm{Ч}_{3}:(3.20)$; для всех $i \in M_{2}$ и $j \in \widetilde{M}_{2}$

$$
0 \leqslant i \leqslant \frac{r}{\ln n}, \quad 0 \leqslant j \leqslant \frac{r}{\ln n} .
$$

При выполнении (3.20) можно воспользоваться неравенством (2.14) и установить

$$
\Gamma_{t, k}^{\omega_{\alpha}} \geqslant C_{r}^{t-1}\left(s^{(\alpha)}+\widetilde{s}^{(\alpha)}\right)
$$

для всех $t \in\{2, \ldots, r\}$ и $\alpha=1, \ldots, \Delta$, где $s^{(\alpha)}=\sum_{i \in I_{\omega_{\alpha}}} i, \widetilde{s}^{(\alpha)}=$ $\sum_{j \in J_{\omega_{\alpha}}} j$. В силу (1.3) и (1.4) для $i=1, \ldots, N$ и $\alpha=1, \ldots, \Delta$

$$
\left|\prod_{t=1}^{g_{i}(n)}\left(1-2 p_{i t}\right)^{\Gamma_{t, k}^{\omega_{\alpha}}}\right| \leqslant \prod_{t \in T_{i}}\left(1-2 \delta_{i t}(n)\right)^{\Gamma_{t, k}^{\omega_{\alpha}}} .
$$


K правой части (3.30) можно применить (3.29) так, что

$$
\prod_{t \in T_{i}}\left(1-2 \delta_{i t}(n)\right)^{\Gamma_{t, k}^{\omega_{\alpha}}} \leqslant \exp \left\{-2 \delta_{i}\left(s^{(\alpha)}+\tilde{s}^{(\alpha)}\right)\right\} .
$$

Принимая во внимание (3.28) и равенство $e^{-y}=1-y+O\left(y^{2}\right), 0 \leqslant y<\infty$, получаем для $i=1, \ldots, N, \alpha=1, \ldots, \Delta$ и $n \rightarrow \infty$

$$
\exp \left\{-2 \delta_{i}\left(s^{(\alpha)}+\widetilde{s}^{(\alpha)}\right)\right\} \leqslant 1-2 \delta_{i}\left(s^{(\alpha)}+\widetilde{s}^{(\alpha)}\right)(1+O(\sqrt{\varepsilon})) .
$$

С помощью (1.4), (3.4) и (3.30)-(3.32) находим при $n \rightarrow \infty$

$$
\begin{aligned}
& 2^{-k N}\left|S^{(\Delta)}(n, k ; Q)\right| \leqslant 2^{-k N}(\Delta+1)^{N} \sum_{s=0}^{n-\rho(n)} C_{n-\rho(n)}^{s} \sum_{s_{*}=0}^{s} R_{1}^{s-s_{*}} \\
& \times\left(\sum_{\sum_{i \in M_{2}}} \frac{s !}{\left(s-s_{*}\right) !} \prod_{i \in M_{2}} i !\right) \sum_{\substack{s_{*}^{\prime}=0_{1} \\
s^{\prime}+s \geqslant 1}}^{\rho(n)} C_{\rho(n)}^{s^{\prime}} \sum_{\tilde{s}_{*}=0}^{s^{\prime}} \widetilde{R}_{1}^{s^{\prime}-\widetilde{s}_{*}} \\
& \times\left(\sum_{\sum_{j \in \tilde{M}_{2}} j=\tilde{s}_{*}} \frac{s !}{\left(s^{\prime}-\widetilde{s}_{*}\right) !} \prod_{j \in \widetilde{M}_{2}} j !\right) \\
& \times \exp \left\{-2^{-z+1} \sum_{i=1}^{N} \delta_{i}(1+O(\sqrt{\varepsilon})) \sum_{\alpha=1}^{\Delta}\left(s^{(\alpha)}+\widetilde{s}^{(\alpha)}\right)\right. \\
& \left.\quad+\left(2^{k}-\Delta-1\right) 2^{-z} \sum_{i=1}^{N} \exp \left\{-2 \sum_{t \in T_{i}} \delta_{i t}(n) C_{r}^{t}\right\}\right\} .
\end{aligned}
$$

Если $\Delta<2^{z}-1$, то из (3.33) с учетом $(1.2),(3.7),(3.15)$ и неравенства $\max \left\{s_{*}, \tilde{s}_{*}\right\} \leqslant \sigma(\varepsilon) n / \ln n$ легко получить $(3.13)$. Пусть $\Delta=2^{z}-1$. Тогда, используя $(1.2),(3.7),(3.15),(3.33)$ и очевидное неравенство

$$
\sum_{\alpha=1}^{\Delta}\left(s^{(\alpha)}+\widetilde{s}^{(\alpha)}\right) \geqslant s_{*}+\widetilde{s}_{*}
$$

где

$$
s_{*}=\sum_{i \in M_{2}} i, \quad \widetilde{s}_{*}=\sum_{j_{1} \in \widetilde{M}_{2}} j
$$

находим оценку

$$
\begin{aligned}
2^{-k N} & \left|S^{(\Delta)}(n, k ; Q)\right| \leqslant 2^{m k}(1+o(1)) \\
& \times \sum_{q=0}^{\infty}\left(\left|M_{2}\right|(n-\rho(n)) \exp \left\{-2^{-z+1}(1+O(\sqrt{\varepsilon})) \sum_{i=1}^{N} \delta_{i}\right\}\right)^{q}(q !)^{-1} \\
& \times \sum_{\substack{\tilde{g}=0 . \\
\tilde{q}+q \geqslant 1}}^{\infty}\left(\left|\widetilde{M}_{2}\right| \rho(n) \exp \left\{-2^{-z+1}(1+O(\sqrt{\varepsilon})) \sum_{i=1}^{N} \delta_{i}\right\}\right)^{\tilde{q}}(\widetilde{q} !)^{-1}, \quad(3.34)
\end{aligned}
$$


$n \rightarrow \infty$, для случая

$$
s_{*}+\widetilde{\boldsymbol{s}}_{*} \geqslant 1 \text {. }
$$

Принимая во внимание утверждение 2.5, нетрудно от (3.34) прийти к (3.13).

Покажем, что (3.35) выполняется, если в (3.14) либо $k \in\{1,2\}$, либо $z \in\{k, k-1\}$. Пействительно, если $z=k$ (или $k \in\{1,2\}$ ), то, очевидно, существует $\alpha \in\{1, \ldots, \Delta\}$, для которого $\xi_{\alpha} \leqslant 2$. Если $z=k-1$, то на основании замечания 2.2 убеждаемся в суцествовании такого $\alpha \in$ $\{1, \ldots, \Delta\}$, что $\xi_{\alpha} \leqslant 2$. Далее, соотношения (2.24) и (2.25) позволяют установить, что если $\xi_{\alpha} \leqslant 2$ для некоторого $\alpha \in\{1, \ldots, \Delta\}$, то $s^{(\alpha)}+\widetilde{s}^{(\alpha)} \geqslant$ 1 , следовательно выполняется (3.35).

Значит, для завершения доказательства (3.13) осталось проверить, что (3.13) имеет место при ограничениях $\mathrm{Ч}_{4}$ :

$$
s_{*}+\widetilde{s}_{*}=0,
$$

$\xi_{\alpha} \geqslant 3, \quad \alpha=1, \ldots, \Delta, \quad \Delta=2^{z}-1, \quad 1 \leqslant z \leqslant k-2, \quad 3 \leqslant k<\infty,(3.37)$ и (3.20). Если выполняется $(3.36), \Delta=2^{z}-1$ и $\widetilde{R}_{1}<2^{k-z}-1$, то оценку (3.33) можно переписать с учетом (3.7) и (3.15) в виде

$$
2^{-k N}\left|S^{(\Delta)}(n, k ; Q)\right| \leqslant 2^{m k}(1+o(1))\left(1-2^{z-k}\right)^{\rho(n)}, \quad n \rightarrow \infty .
$$

Принимая во внимание (3.19), получаем (3.13).

Убедимся в справедливости (3.13) при ограничениях $Ч_{4}$ и

$$
\widetilde{R}_{1}=2^{k-z}-1
$$

При выполнении (3.37) и (3.38) множество $\widetilde{M}_{1}$ содержит. согласно утверждению 2.2 не менее трех таких элементов $j_{m_{\nu}} \in \widetilde{M}_{1}, \nu=1,2,3$, что для некоторого $\alpha \in\{1, \ldots, \Delta\}$

$$
\left|\omega_{\alpha} \cap m_{\nu}\right|=2, \quad \nu=1,2,3 ; \quad\left|\omega_{\alpha} \cap(a \cup b)\right|=3
$$

для произвольных $a, b \in\left\{m_{\nu}: \nu=1,2,3\right\}, a \neq b$. Для указанного $\alpha$ в силу (2.11) и (3.39) имеем:

$$
\Gamma_{t, k}^{\omega_{\alpha}} \geqslant \gamma_{t}^{\{a \cup b\}}, \quad t \in\{2, \ldots, r\} .
$$

Оценивая правую часть (3.40) с помощью (2.16), находим

$$
\gamma_{t}^{\{a \cup b\}} \geqslant t^{-1} j_{*}\left(j^{*}-2^{-1}\left(j_{*}-1\right)\right) C_{\left(j^{*} / 2\right)+\left(3 j_{*} / 4\right)-(5 / 4)}^{t-2}
$$

при условии, что $j_{*} \geqslant t$, где $j_{*}=\min \left\{j_{a}, j_{b}\right\}, j^{*}=\max \left\{j_{a}, j_{b}\right\}$. Если $j_{*}>$ $[\sqrt{\varepsilon} \varphi(n)]$, то неравенство $j_{*} \geqslant t, t \in\{2, \ldots, r\}$, заведомо выполняется для $0<\varepsilon<1$, и поэтому из (3.40) и (3.41) следует для указанных $j_{*}$ и $t$ при $n \rightarrow \infty$

$$
\Gamma_{t, k}^{\omega_{\alpha}} \geqslant \varepsilon(2 t)^{-1} \varphi^{2}(n) C_{(5 \sqrt{\varepsilon} \varphi(n) / 4)-(5 / 4)}^{t-2}
$$


что противоречит (3.3) при достаточно малом $\varepsilon>0$ и $t \in\{2, \ldots, r\}$. Таким образом, при ограничениях (3.37) и (3.38) по крайней мере один элемент $j_{*}$ множества $\widetilde{M}_{1}, j_{*} \in \widetilde{M}_{1}$, удовлетворяет неравенству $j_{*}<$ $[\sqrt{\varepsilon} \varphi(n)]$. Поэтому при ограничениях $Ч_{4}$ и $(3.38)$ в условиях теоремы получаем при $n \rightarrow \infty$

$$
2^{-k N}\left|S^{(\Delta)}(n, k ; Q)\right| \leqslant \exp \{(\sigma(\varepsilon)+o(1)) \rho(n)\}\left(1-2^{z-k}\right)^{\rho(n)} \longrightarrow 0 .
$$

Соотношение (3.42) доказывает (3.13) в предположениях $\mathrm{Ч}_{4}$ и (3.38).

Анализируя ограничения $\mathrm{Ч}_{i}, i=0,1, \ldots, 4$, нетрудно убедиться в том, что полученное при этих ограничениях соотношение (3.13) справедливо для всех значений параметров $s, s^{\prime}, i, j(i \in I, j \in J)$ суммирования в (2.22), при которых неравенство (3.3) имеет место для $\Delta \geqslant 1$. Из (3.2), (3.5) и (3.13) немедленно следует (3.1) для $k=1,2, \ldots$ Так как $\lambda^{k}$ есть $k$-й, $k \geqslant 1$, факториальный момент для распределения Пуассона с параметром $\lambda$ и выполняется (3.1), то по известной теореме (см., например, $\left[7\right.$, с. 260]) распределение случайной величины $\nu_{n}$ сходится при $n \rightarrow \infty$ к распределению Пуассона с тем же параметром $\lambda$. Соотношение (1.7) установлено. Теорема доказана.

\section{СПИСОК ЛИТЕРАТУРЫ}

1. Kovalenko I. N., Levitskaya A. A. Stochastic properties of systems of random linear equations over finite algebraic structures. - В сб.: Вероятностные методы дискретной математики./Под. ред. В. Ф. Колчина и др. (Труды третьей Петрозаводской конференции. Петрозаводск, 12-15 мая, 1992.) Москва/Утрехт: TBП/VSP, 1993, c. $64-70$.

2. Балакин $Г$. В. Графы систем двучленных уравнений с булевыми неизвестными. - Теория вероятн. и ее примен., 1995, т. 40, в. 2, с. 241-259.

3. Копытчев $B$. A. О распределении числа решений случайных заведомо совместных систем уравнений. - Теория вероятн. и ее примен., 1995, т. 40, в. 2, с. 430437.

4. Масол В. И. Пуассоновские теоремы для предельного распределения числа решений системы нелинейных случайных булевых уравнений. - В сб.: Вторая Всероссийская школа-коллоквкум по стохастическим методам. Тезисы докладов. М.: ТВП, 1995, с. 95-96.

5. Экдрюс Г. Теория разбиений. М.: Наука, 1982, 256 с.

6. Masol V.I. Moments of the number of solutions of a system of random. Boolean equations. - Random Operators and Stochastic Equations, 1993, v. 1, № 2, p. 171179.

7. Сачков $B$. Н. Введение в комбинаторные методы дискретной математики. М.: Наука, 1982, 384 c.

Поступила в редакцию 8.IV.1996 